\title{
Decompensated Heart Failure in Pregnancy
}

\author{
John Anthony ${ }^{1}$ and Karen Sliwa2,3 \\ 1. Division of Obstetrics and Gynaecology Groote Schuur Hospital, University of Cape Town, Cape Town, South Africa; \\ 2. Hatter Institute for Cardiovascular Research in Africa, Department of Medicine, Faculty of Health Sciences, University of Cape Town, Cape Town, South Africa; 3 . \\ Soweto Cardiovascular Research Group University of the Witwatersrand, South Africa
}

\begin{abstract}
Heart disease is a common cause of morbidity and mortality during pregnancy. Symptoms and signs of heart failure in a pregnant woman are an indication for urgent assessment to establish a diagnosis and appropriate management. This is best accomplished through a multidisciplinary approach in which both cardiologists and obstetricians need to participate in order to provide expert counselling and care in pursuit of safe motherhood. Congenital heart disease, although common, once corrected is an unusual source of complications, which are more likely to develop as a consequence of ventricular failure, pulmonary hypertension and aortic arch disease. Rheumatic valvular heart disease is a challenge because of the need for anticoagulation during pregnancy and the risk of sepsis associated with childbirth. This review outlines a contemporary approach to heart failure presenting during pregnancy.
\end{abstract}

\section{Keywords}

Pulmonary oedema, heart failure, pregnancy, maternal mortality, cardiomyopathy

Disclosure: The authors have no conflicts of interest to declare.

Received: 14 August 2015 Accepted: 21 October 2015 Citation: Cardiac Failure Review, 2015;1(2):20-6 DOI: 10.15420/cfr.2015:24:2

Correspondence: Karen Sliwa, Hatter Institute for Cardiovascular Research in Africa, Faculty of Health Sciences, University of Cape Town, Private Bag X3 7925

Observatory, Cape Town, South Africa. E: Karen.Sliwa-Hahnle@uct.ac.za

'Heart failure' is a term that may be loosely or precisely defined. The development of pulmonary oedema does not necessarily indicate a cardiac cause and of the cardiac causes for pulmonary oedema, not all can be attributed to left ventricular failure. ${ }^{1}$ The majority of women developing symptoms and signs of heart failure during pregnancy have no known pre-existing cardiomyopathy. This article describes the cardiac causes of pulmonary oedema presenting in pregnancy with reference to other differential diagnoses.

\section{Significance of Heart Failure as a Cause for Mortality and Morbidity}

Cardiac disease complicating pregnancy is categorised as an 'indirect' cause of maternal mortality, meaning that it is unrelated to any complication of the pregnancy itself. ${ }^{2}$ Medical and surgical disorders that cause indirect maternal deaths are a diverse group of diseases that include various medical, surgical and infective causes of maternal illness. Globally, indirect causes of maternal mortality account for a quarter of the deaths reported by industrialised countries with even higher rates in southern Asia and sub-Saharan Africa: 29.3 and $28.6 \%$, respectively. ${ }^{3}$

Accurate epidemiological data have been reported in a few countries that have pursued statutory notification of all maternal deaths. In the UK, the rates of maternal mortality have been recorded in single digit figures per 100,000 live-births. ${ }^{4}$ By contrast, South African data (also accumulated by means of statutory, confidential enquiry) reflect rates of 179 per 100,000 live-births. ${ }^{5}$ The South African data are dominated by HIV-related mortality, accounting for $40 \%$ of all deaths. However, medical and surgical disorders, representing the bulk of the remaining indirect causes of death account for $8.8 \%$ of mortality, rendering this the fourth most-common cause of mortality, ranking only behind HIV, haemorrhagic deaths and deaths due to hypertension. ${ }^{2}$ Significantly, the category of 'medical and surgical' disorders contains a preponderance of cardiac deaths, which are the single most-common entity in this group accounting for $36.5 \%$ of mortality with ventricular failure responsible for just over half these deaths.

The data from the UK identify indirect deaths attributable to cardiac disease as a cause for $20 \%$ of the reported mortality, being the single commonest cause of indirect death and the commonest overall cause for maternal death. ${ }^{6}$ In this series, nearly a quarter of the cardiac deaths were diagnosed to be due to cardiomyopathy. The preponderance of deaths due to ventricular failure is further underlined by deaths that occurred outside the window in which postpartum cases are usually reported. 'Late' deaths due to peripartum cardiomyopathy often remain unreported and the true estimate of mortality related to ventricular failure during pregnancy may be underestimated by up to $50 \%$. The data from the UK are diagnostically specific, allowing characterisation of different kinds of ventricular failure into those due to peripartum cardiomyopathy, dilated cardiomyopathy of various kinds and right ventricular arrhythmogenic cardiomyopathy.

Genetic and social variations do exist within individual communities giving rise to disparities in the incidence of ventricular failure due to peripartum cardiomyopathy seen in multicultural societies. Hence, in the US, large disparities are seen with $1: 1,421$ in African Americans developing ventricular failure in pregnancy compared with an incidence of $1: 2,675$ Asians, $1: 4,075$ in Caucasians and $1: 9,861$ in people of Hispanic origin.? 
Although the relative prevalence of cardiac disease varies between different countries, it remains a common cause of maternal death in both developing countries and industrialised societies with ventricular failure being the single most- common mechanism of death.

\section{Cardiovascular Physiology During Pregnancy}

The physiological demands of pregnancy are partly met through changes in cardiovascular physiology, which has to accommodate the extra metabolic demands presented by the foetus and other organ systems required to perform an augmented physiological role. Hence, the increase in uterine size and activity together with the blood flow into the choriodecidual space are a significant component of the extra cardiovascular work during pregnancy accounting for $12 \%$ of the total cardiac output in a term pregnancy. ${ }^{.}$The kidneys and the skin, in particular, have augmented perfusion to disperse heat and allow the retention of sodium and water during pregnancy. The increased blood volume secondary to hyperaldosteronism allows an increase in cardiac output mediated by increased stroke volume and heart rate; the cardiac output rises in the first trimester peaking towards the end of the second trimester at between 3.5 and 6 litres per minute (which is $30-50 \%$ higher than non-pregnant values). 9.10 The increased cardiac output is discharged into a dilated peripheral systemic circulation that shows a falling systemic vascular resistance from the first trimester onwards. The extent of the adaptation is such that the arteriovenous oxygen difference falls during early pregnancy and rises towards pre-pregnancy levels by the end of the pregnancy. Both left and right ventricles show evidence of increased mass, volume and enddiastolic volume during pregnancy. These changes reflect the increase in cardiac output and intravascular blood volume and reverse after delivery. ${ }^{11}$ These adaptations are summarised in Figure 1 below.

\section{Aetiology and Pathophysiology of Heart Failure in Pregnancy}

Cardiovascular disease complicating pregnancy may be considered in groups including those attributable to increased vascular resistance, diseases of the aortic root, heart disease itself due to either obstruction, ventricular failure or congenital abnormalities of the heart and proximal vasculature. Three pregnancy-specific causes of heart failure are identifiable (pre-eclampsia, peripartum cardiomyopathy and amniotic fluid embolism) together with all the non-pregnancyrelated causes of heart failure that may become co-morbid diseases complicating pregnancy.

\section{Increased Vascular Resistance \\ Pre-eclampsia}

Pre-eclampsia commonly results in pulmonary oedema that, together with cerebrovascular haemorrhage, has been identified as the dominant cause of hypertensive maternal mortality by a South African confidential enquiry. ${ }^{2}$ Pregnancy and pre-eclampsia are usually associated with a hyperdynamic circulation and enhanced left ventricular contractility. ${ }^{12}$ In the case of pre-eclampsia, increased systemic vascular resistance may increase the filling pressures in the left atrium and, together with intravenous fluid administration, will increase the likelihood of developing pulmonary oedema. The direct cardiac contribution to the development of pulmonary oedema is usually due to diastolic dysfunction..$^{13}$ The left ventricle tolerates an intravenous fluid load poorly showing a rapid rise in left-sided filling pressures without any similar observable changes in the right heart. 14,15 Occasionally mildly impaired systolic function will be identified in severe pre-eclampsia, although this is usually transitory.
Figure 1: Summary of Physiological Changes Occurring During Pregnancy

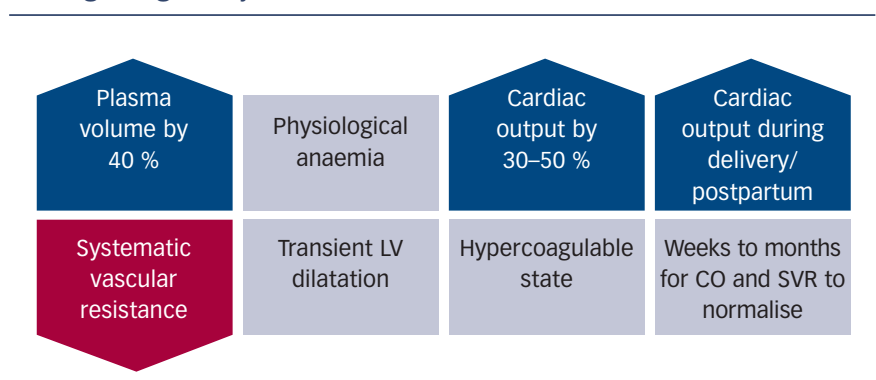

$C O=$ cardiac output; $L V=$ left ventricle; $S V R=$ systemic vascular resistance.

\section{Hypertensive Cardiomyopathy}

Chronic hypertension complicating pregnancy in the absence of superimposed pre-eclampsia is not clearly associated with adverse maternal outcome ${ }^{16,17}$ Chronic hypertension prior to pregnancy is, however, increasing in frequency due to the worldwide obesity epidemic and is prevalent in $3 \%$ of all US pregnant women. ${ }^{18}$ Chronic hypertension leads to increased frequency of preeclampsia (17-25\% versus $3-5 \%$ in the general population), as well as placental abruption, foetal growth restriction and preterm birth. ${ }^{18}$

Hypertensive CMO may lead to diastolic dysfunction; together with the increase in pregnancy preload, this may predispose some patients to mild pulmonary oedema, usually at the time that plasma volume expansion reaches peak values at 32 to 34 weeks' gestation..$^{19,20}$

\section{Pulmonary Hypertension and Right Heart Failure}

The symptoms suggestive of pulmonary hypertension are those exertional dyspnoea with everyday activities. Weakness and recurrent syncope are also common. These symptoms may be followed by signs consistent with right heart failure (increased jugularis venous pressure [JVP], loud second heart sound/P2, hepatomegaly and peripheral oedema with clear lung fields). The causes of pulmonary hypertension are grouped into primary (idiopathic) and secondary causes and have been classified in a consensus statement. ${ }^{21}$ The secondary causes are divided into pre- and post-capillary pulmonary hypertension. These are typically due to lung parenchymal disease or the various cardiac causes of increased left-sided filling pressures. Pregnant women with primary pulmonary hypertension are at particular risk of acute right-sided heart failure after delivery and may have a sudden death. The mechanism of acute deterioration has not been elucidated. ${ }^{22}$

\section{Diseases Affecting the Aortic Root}

This can be broadly classified into medial disorders with a risk of dissection such as Marfan's syndrome, inflammatory diseases of the aorta, usually Takayasu's arteritis and atherosclerotic disease. Marfan's and Takaysu's syndromes are the two common aortic arch diseases that may present during pregnancy. The presenting features of Takayasu's disease are predominantly hypertension, although some patients may present with heart failure. ${ }^{23}$ Arterial dissection and acute aortic regurgitation are more likely acute presentations of Marfan's syndrome during pregnancy, especially in those with a dilated aortic root. ${ }^{24}$

\section{Cardiac Disease - Ventricular Failure}

Cardiomyopathy is generally the commonest cause of mortality developing during or after pregnancy. ${ }^{25,26}$ 


\section{Peripartum Cardiomyopathy}

This is a well-characterised disease developing in previously well women who develop left ventricular systolic dysfunction with an ejection fraction of less than $45 \%$ during the last month of pregnancy or within 6 months of delivery. ${ }^{27}$ The European Society of Cardiology definition is less specific, stating that it is an idiopathic cardiomyopathy presenting with left ventricular failure towards the end of pregnancy or in the months following pregnancy for which no other cause can be found. Typically, but not inevitably, this is a dilated cardiomyopathy that develops in the absence of any identifiable cause of heart failure, including genetic cardiomyopathies. The pathophysiology is incompletely understood but a $16 \mathrm{kDa}$ cathepsin cleavage product of prolactin has been identified as a possible mechanism based upon the induction of myocyte apoptosis. ${ }^{27,28}$ Other identified mechanisms include inflammatory changes that induce oxidative stress and the development of autoimmunity to proteins of cardiac origin. ${ }^{29}$ The latter mechanism may be the result of cardiac injury rather than a causative mechanism. Other investigators have pointed out the association between peripartum cardiomyopathy, preeclampsia and multiple pregnancy, which are all linked to an imbalance in angiogenic and antiangiogenic factors raising the possibility that this mechanism may also be one of the pathways leading to the development of cardiomyopathy. ${ }^{30}$

\section{Hereditary Cardiomyopathies}

Hereditary cardiomyopathies are grouped into hypertrophic (HOCM), dilated (DCMO) and right ventricular arrhythmogenic cardiomyopathy (RVCMO), broadly representing genetic mutations in the cardiac sarcomere, the myocyte and desmosomes, respectively. ${ }^{31}$ Of these conditions, the hypertrophic form of the disease is well tolerated in pregnancy providing there are no pre-pregnancy symptoms related to HOCM. ${ }^{32,33}$ The diagnosis of HOCM is phenotypic, based upon the increase in left ventricular wall diameter that cannot be accounted for by co-existing hypertension or valvular disease. Dilated cardiomyopathy is more usually due to conditions other than genetic disease, which account for about one-third of such cases. There are no specific data detailing the pregnancy outcomes in women with genetic DCMO. In general, the outcome of pregnancies complicated by the presence of a DCMO is predicted by the severity of pre-pregnancy symptoms. Right ventricular arrhythmogenic $\mathrm{CMO}$ is associated with a risk of sudden cardiac death, which is not altered by the occurrence of pregnancy. ${ }^{34}$

\section{Drug-induced CMO}

The drugs associated with induced CMO consist of a long list of which certain agents are more notable than others. In particular, alcohol, cocaine, amphetamines, methamphetamine, catecholamines, ephedrine, zidovudine, chloroquine, cyclophosphamide and certain antimitotic drugs. While the use and abuse of these agents may take place during pregnancy they are rarely identified as a cause of acute heart failure

\section{Autoimmune and Infective $\mathrm{CMO}$}

Myocarditis on the basis of viral infection or autoimmunity commonly leads to dilated cardiomyopathy with chronic heart failure. Acute damage caused by viral infection may be followed by the exposure of sequestered intracellular antigens that perpetuate immune damage via both the innate and adaptive immune systems. ${ }^{35}$

\section{Amniotic Fluid Embolism}

Amniotic fluid embolism is a complication of labour with an acute onset of a syndrome characterised by cardiovascular collapse, pulmonary oedema, seizure activity and a bleeding diathesis. ${ }^{36}$ The mechanisms are poorly understood but the release of large amounts of amniotic fluid and fetal squames into the maternal circulation triggers acute pulmonary hypertension followed by left ventricular failure requiring inotropic support for a protracted period of time. Arrhythmias are also common.

\section{Ischaemic Heart Disease}

This is a rare complication of pregnancy that usually presents with symptoms of ischaemia rather than heart failure. It is estimated to affect one in 10,000 pregnancies. The major risk to the mother is that of mortality secondary to acute myocardial infarction during pregnancy; however, the recent literature suggests that acute coronary syndromes are unusual and the mortality risk may not be as high as previously suspected. ${ }^{25}$ Apart from the usually identified dyslipidaemia giving rise to coronary artery disease, anomalous origins of the coronary arteries, coronary artery dissection, severe aortic stenosis and hypertrophic cardiomyopathy may all present with ischaemic symptoms and signs. Deaths due to coronary artery disease are unusual in European countries and have not been recorded in African maternal mortality reviews either.

\section{Cardiac Disease - Valvular Lesions}

Post-rheumatic valvular heart disease may lead to pulmonary oedema during pregnancy for a number of reasons. The haemodynamic consequences of pregnancy alone may precipitate pulmonary oedema on the basis of an increasingly hyperdynamic circulation and increased plasma volume. The increased plasma volume peaks at 34 weeks' gestation with an acute rise immediately after delivery of the baby and placenta. The increased pulse rate of pregnancy, further aggravated by the pain of labour, means that left ventricular filling times diminish increasing the risk of heart failure in women with stenosed mitral valve disease.

In general, regurgitant lesions, such as mitral and aortic valve regurgitation, are better tolerated in pregnancy compared with stenotic lesions. ${ }^{37}$ Limited data exist on mixed valve lesions or multiple lesions affecting several valves due to rheumatic heart disease. In addition, arrhythmias and various co-morbidities such as anaemia, hypertension and thyroid disease all contribute to a greater risk of pulmonary oedema in patients with valve disease. In the European registry, valvular heart disease were commonly diagnosed for the first time during pregnancy and led to a greater risk of maternal mortality than heart disease caused by congenital defects. ${ }^{25}$ Heart failure, arrhythmias and obstetric haemorrhage were all noted to be complications of valvular disease.

\section{Cardiac Disease - Congenital Abnormalities of the Heart and Proximal Vasculature}

Uncorrected congenital heart disease complicating pregnancy is a rare event. Generally operated congenital heart disease women have uncomplicated pregnancies providing their pre-pregnancy effort tolerance is good. ${ }^{38}$ Of the uncorrected lesions, Marfan syndrome with a dilated aortic root and Eisenmenger syndrome carry the greatest risk of mortality due to dissection or heart failure. Congenitally stenosed valves, cyanotic disease in the absence of pulmonary hypertension, a systemic right ventricle or Fontan circulation carry a moderate risk of heart failure whereas the septal defects and repaired co-arctation are regarded as low-risk lesions. ${ }^{39}$

\section{Clinical Presentation}

The clinical presentation of acute heart failure in pregnancy may be divided into clinical signs of left, right or bi-ventricular heart failure. 
Dyspnoea is the most common phenotypic expression of all kinds of acute heart failure. There are many causes for dyspnoea and a diagnosis cannot be made without a thorough examination and investigation.

\section{Causes and Nature of Dyspnoea}

Dyspnoea and tiredness are often terms used interchangeably by pregnant women. True shortness of breath may be experienced in a normal pregnancy as a consequence of increased respiratory drive mediated by the effects of progesterone. ${ }^{40}$ Physiological over-breathing is a pregnancy adaptation accelerating the excretion of carbon dioxide allowing the foetus a greater gradient down which to offload carbon dioxide and to maintain acid-base homeostasis. Dyspnoea aggravated by exertion together with other symptoms suggestive of cardiac failure, such as orthopnoea, paroxysmal nocturnal dyspnoea, blackouts and palpitations, clearly indicates the need for further assessment. Fett has suggested that women could monitor their own symptoms, which would allow earlier recognition of dyspnoea in those at risk of heart failure, and has even suggested a scoring system to facilitate this process. $^{41}$

\section{Clinical Signs}

Pregnancy may mimic some signs of heart disease. ${ }^{42} \mathrm{~A}$ bounding full pulse and mildly elevated jugular venous pressure is a consequence of increased plasma volume and a hyperdynamic circulation. Worsening peripheral oedema is also a physiological consequence whereby extravascular fluid is allowed to accumulate towards the time of delivery when haemorrhage following childbirth may necessitate physiological transfusion of fluid stored in the tissues into a depleted intravascular compartment. Palmar erythema is commonly observed in the absence of liver disease and reflects increased skin perfusion. The heart rate is mildly elevated with arrhythmias including sinus arrhythmia occurring more commonly. ${ }^{43}$ Examination of the praecordium may show an apex beat displaced to the sixth intercostal space beyond the mid-clavicular line. Systolic flow murmurs are commonly heard at the left sternal edge, caused by increased flow through the aortic and pulmonary valves. ${ }^{44} \mathrm{~A}$ mammary souffle may also be heard from increased flow through the internal thoracic vessels. A third heart sound can also be heard in a normal pregnancy.

When assessing a pregnant woman with a history suggestive of heart failure, the findings that should prompt further investigation if they include any evidence of cyanosis or clubbing, a resting tachycardia, any arrhythmia, a collapsing pulse, hypertension or hypotension, any tachypnoea or other clinical signs of respiratory distress, a pulsatile and elevated JVP, any cardiac murmur that is heard in diastole, any cardiac murmur that is pansystolic or radiates beyond the left sternal edge, any loud murmur with an associated thrill or any clinical signs of pulmonary oedema.

\section{Investigations}

Chest X-ray and electocardiogram (ECG) are needed in all circumstances. The pregnancy physiological changes may result in increased pulmonary vascular markings and the enlarging uterus may displace the diaphragm upwards, mitralising the cardiac shadow. The ECG may show ST segment abnormalities suggestive of ischaemia as well as supraventricular arrhythmias in a normal pregnancy. ${ }^{42}$

Echocardiography in pregnancy has the same utility as it does in nonpregnant women and ought to be routinely utilised in anyone with symptoms suggestive of cardiac disease.

\section{Assigning Severity of Risk}

Traditionally, the New York Heart Association Classification has been used to grade degrees of exertional dyspnoea. ${ }^{45}$ Although still in use, epidemiological data have been combined with the presence or absence of symptoms into a modified World Health Organization (WHO) classification aimed at defining the risk of adverse outcomes (morbidity and mortality) in pregnant women. ${ }^{39,46}$ Class I cardiac disease is not associated with any risk and includes uncomplicated mild pulmonary stenosis, patent ductus arteriosus and mitral valve prolapse together with repaired septal defects and isolated supra ventricular and ventricular ectopic beats. Class II diseases include all other arrhythmias, repaired tetralogy of Fallot and operated septal defects that are all associated with a small increase in risk. WHO category II-II disease in which there is a significantly increased risk of adverse outcomes, include all forms of cardiomyopathy, prosthetic heart valves, Marfan syndrome with a graduated risk according to the extent of aortic dilatation, cyanotic heart disease, fontan circulation and a systemic right ventricle. WHO IV, where the risk of adverse outcome is high enough to consider pregnancy contraindicated, includes pulmonary hypertension, severe symptomatic aortic or mitral stenosis, previous peripartum cardiomyopathy with residual ventricular impairment, severe left ventricular failure (ejection fraction less than $30 \%$ ) and aortic disease (either coarctation or Marfan's syndrome with dilated aortic root of more than $45 \mathrm{~mm}$ ).

The WHO classification underscores the importance of expert counselling and joint care shared between cardiologists and obstetricians for all women with WHO class III and IV disease. ${ }^{26}$ Combined assessment clinics are commonly centralised in large teaching hospitals in both industrialised and developing countries with successful outcomes documented when treating women with WHO stage III and IV disease.

\section{Differential Diagnosis}

Heart failure in pregnancy needs to be differentiated from other causes of respiratory distress.

Non-cardiogenic pulmonary oedema must be distinguished from cardiovascular causes of pulmonary oedema. Adult respiratory distress syndrome is an uncommon complication of pregnancy and usually the sequel to pre-existing lung pathology, such as infection. ${ }^{47} \mathrm{~A}$ diagnosis of non-cardiogenic pulmonary oedema cannot be made before thorough investigation of the cardiovascular system to eliminate any cardiac contribution to the development of pulmonary oedema.

Pulmonary parenchymal disease arising from infection as well as interstitial lung disease may present during pregnancy. Although the clinical and radiological signs may help to distinguish one cause of respiratory distress from another, the clinical differential diagnosis of infection compared with pulmonary oedema may sometimes be difficult to resolve. Pulmonary thromboembolism is often also considered in the differential diagnosis of acute respiratory distress during pregnancy. Pregnancy is a pro-coagulant condition and thromboembolism is a common cause of both acute morbidity and mortality; the clinical diagnostic criteria for thromboembolism are poorly predictive of the condition and consequently special investigation is mandatory when thromboembolism is part of the differential diagnosis. ${ }^{48}$

Metabolic disorders can also lead to tachypnoea with various causes of acidosis in question. Diabetic ketoacidosis is seen commonly whereas lactic acidosis may result from the adverse effects of certain drugs. 


\section{Clinical Evaluation}

Sub-diaphragmatic pathology associated with any pain may cause rapid shallow respiration, which may be mistaken for respiratory distress. Pre-eclampsia complicated by ischaemic liver necrosis (the syndrome of Haemolysis, Elevated Liver enzmes and Low Platelets otherwise known as the HELLP syndrome) is one such common cause for this presentation. ${ }^{49}$

Addressing the differential diagnosis requires a comprehensive history and clinical examination followed by relevant investigations with the most-common differential being between infection, pulmonary oedema and thromboembolism.

\section{Management Principles}

\section{Drug therapy}

The pharmacological management of pregnant women with cardiac lesions including the management of heart failure and the possible effect of the medication on the foetus has recently been summarised in a dedicated book. ${ }^{50}$

\section{Diuretics}

Diuretics are the first line of treatment for most pregnant women with heart failure..$^{51}$ The increased preload associated with pregnancy is part of the mechanism by which a hyperdynamic circulation develops. Women with normal ventricular function respond to increased preload with increased output. That may not happen when the left ventricular contractility is abnormal. Reducing preload will diminish left-sided filling pressures as well as pulmonary capillary pressures allowing the resorption of pulmonary interstitial fluid. There is a theoretical concern that using treatment that partially reverses or limits the physiological changes of pregnancy may adversely affect the pregnancy outcome by limiting the necessary increase in uterine and, therefore, placental perfusion. However, the foetuses of women with cardiac disease are prone to develop restricted growth and discerning the effects of treatment from those of the disease (low cardiac output due to ventricular failure) is difficult outside the context of controlled studies. There is no evidence that diuretics are an independent risk factor for foetal growth restriction and the use of diuretics in circumstances where the mother becomes symptomatic on the basis of increased preload complicating left ventricular dysfunction justifies the use of diuretic therapy as first-line treatment.

Whereas diuretic therapy may be useful when ventricular dysfunction is the cause of pulmonary oedema, in other circumstances the use of diuretics may be less beneficial. Pre-eclampsia when presenting as acute severe disease, does so with elevated vascular resistance and often some degree of left ventricular diastolic dysfunction..$^{14}$ In this setting, afterload reduction using parenteral vasodilators is a preferable treatment because falling vascular resistance allows an increase in left ventricular stroke volume and cardiac output with a secondary decrease in left-sided filling pressures.52 Diuretic therapy, by contrast, may deplete an already contracted intravascular volume with no reduction in systemic vascular resistance. The diversity of underlying mechanisms giving rise to pulmonary oedema makes clinical discrimination and rational therapy very difficult when all causes have a common phenotype, namely pulmonary oedema. Only patients with access to invasive haemodynamic monitoring or ready access to echocardiography are likely to be managed consequentially based upon a recognised haemodynamic subset.53,54

Nitroglycerine as a combined arterial and venous vasodilator may have a role to play especially in the management of acutely ill pregnant women admitted to intensive care. This is especially so in women who have hypertension due to pre-eclampsia complicated by both left ventricular systolic failure and simultaneous renal failure. Increasing venous capacitance under these circumstances may be beneficial.

The heterogeneity of pathophysiological mechanisms related to the development of pulmonary oedema may also confound epidemiological investigations that use the phenotype alone to recruit patients into randomised studies.

\section{Angiotensin Converting Enzyme Inhibitors}

This class of drugs includes the angiotensin receptor blockers and converting enzyme inhibitors. ${ }^{55}$ The drugs intercept the reninangiotensin-aldosterone axis leading to natriuresis, reduced intravascular volume and vasodilation. Whereas these drugs are standard forms of treatment in non-pregnant individuals with heart failure, pregnancy is a state of physiological hypereninism in which the use of these drugs are relatively contraindicated. ${ }^{42}$ Apart from the effects of the renin-angiotensin-aldosterone axis in regulating central haemodynamics, the mechanism may also be important in controlling specific perfusion of certain organs, notably the uterus and the kidney. ${ }^{56}$ Babies delivered by mothers treated with angiotensin converting enzyme (ACE) inhibitors during pregnancy are at increased risk of neonatal renal failure and for this reason the drugs are viewed as being contraindicated. ${ }^{57}$

The breastfeeding mother can safely take ACE inhibitors with sufficient data available to establish the safety of this practice.

\section{Beta-blockers}

Beta-adrenergic receptor blockade slows the heart rate and allows greater filling during diastole. The use of beta-blockers in patients with systolic dysfunction and an ejection fraction $<40 \%$ has been associated with improved symptoms and survival. ${ }^{58}$ During pregnancy, data pertaining to the use of beta-blockers in women with hypertension has shown an association between the use of beta-blockers and intrauterine growth restriction and increased perinatal mortality. ${ }^{59}$ This does not constitute a contraindication to the use of beta blockade during pregnancy when the mother's life is at risk and where both the underlying maternal disease and the treatment can cause adverse perinatal outcome. Epidemiological data identifying risks associated with the use of beta blockade are all confounded by an inability to discriminate between the perinatal effects of the disease and the effects of the treatment.

\section{Spironolactone}

This is a potassium-sparing aldosterone antagonist that is a mild diuretic. The effects of spironolactone are synergistic with those of other diuretics and co-treatment with spironolactone results in a significantly reduced risk of mortality among those who have heart failure. ${ }^{60}$ In pregnancy, the anti-androgen effects of spironolactone, together with some evidence of teratogenesis in the rat-model, has meant that the drug should not be used during pregnancy. In the puerperium, the treatment of the mother should proceed according to the needs of the adult. The use of spironolactone in these circumstances results in less $<1 \%$ of the drug passing from mother to child in the breastmilk.

\section{Bromocriptine}

The suppression of lactation and prolactin secretion may be important in specific circumstances. Where the diagnosis of peripartum 
cardiomyopathy has been made, research suggests that one of the mechanisms causing this condition may be the presence of a $16 \mathrm{kDa}$ cathepsin-cleavage product of prolactin with apoptotic effects on the myocardium. ${ }^{28}$ In these circumstances, given the poor prognosis of peripartum cardiomyopathy, the use of bromocriptine to suppress lactation is a reasonable treatment, based on limited data, although consensus is still lacking for this intervention. A multicentre randomised study is in progress to examine the effects of bromocriptine on left ventricular function in women who present with peripartum cardiomyopathy. ${ }^{61}$

\section{Managing Arrhythmias}

Sudden cardiac death accounts for up to $50 \%$ of the deaths associated due to heart failure. The most common arrhythmia encountered is atrial fibrillation, which may be treated with beta-blockers and (if necessary) digoxin. Ventricular arrhythmias causing sustained ventricular tachyarrhythmia may be diagnosed more accurately when implantable devices are used for continuous monitoring. The management of these patients may include the use of amiodarone and implantable cardioverter defibrillators. ${ }^{62}$

\section{Inotropic Support and Assist Devices}

Inotropic support may be a necessary treatment in acutely ill women with severely impaired ventricular function. Persistent hypotension due to refractory heart failure may benefit from temporary inotropic support while reversing the pregnancy preload and treating underlying hypertensive disease. There is no randomised data characterising the management of pregnant women needing this intervention and the treatment will always be based upon the clinical assessment of women needing intensive care. Assist devices are a potential adjunct in the management of intractable heart failure where they may bridge the gap between the initial presentation of the patient and access to cardiac transplantation services. Again, there is limited evidence. ${ }^{62}$

\section{Treating Reversible Factors}

Factors that may aggravate heart failure increasing the heart rate include anaemia, overt or occult infection and hyperthyroidism. These factors should be considered and managed, each on their own merits. Hyperthyroidism in relationship to pregnancy may be a particularly under diagnosed condition in the puerperium. ${ }^{63}$ In the first year after delivery, postpartum thyroiditis may affect up to $5 \%$ of women. This is often subclinical and undiagnosed.

Of the infections, urinary infection is a frequent cause of sepsis during pregnancy and together with the risk of genital tract sepsis after delivery, needs to be recognised and treated in good time.

\section{Supportive Measures}

The critical care measures taken in women with decompensated heart failure during pregnancy are all standard measures that include the maintenance and monitoring of oxygenation. Pharmacological intervention is detailed in the preceding section and immediate management is usually followed by the necessary investigations to establish the diagnosis.

During pregnancy, the hospitalised pregnant woman with heart failure is in need of thromboprophylaxis, which should be given in the form of subcutaneous low molecular weight heparin as a daily dose; which should be given in a prophylactic dose, typically $40 \mathrm{mg}$ of enoxaparin. ${ }^{64}$
The obstetric management of a woman with decompensated heart failure in pregnancy depends upon an assessment of the degree to which the pregnancy physiology is contributing to the development of heart failure and the risks of continuing with the pregnancy. These factors are a matter of professional judgement, which take into account the specific risks to the mother, the consequence of preterm birth for the baby and the opinion of the mother and her partner in reaching a decision about continuation or termination of the pregnancy. Most cardiac causes for pulmonary oedema would be subject to a trial of treatment with medical therapy before considering ending the pregnancy. Pulmonary oedema developing in pre-eclamptic mothers is always an indication for ending the pregnancy because of the high fatality rate and clear epidemiological association between the onset of pulmonary oedema and the risk of maternal mortality.

\section{The Mode of Delivering the Foetus}

The option of operative delivery or vaginal birth needs to be considered. The haemodynamic consequences of labour are a further increase in left ventricular workload, a pain-mediated increase in catecholamines and an acute increase in intravascular blood volume at the point of delivery, offset to some extent by blood loss at the time of delivery. The second stage of labour is a time during which the mother may need to valsalva, the cardiovascular effects of which may be limited by means of assisted vaginal delivery using either forceps or a vacuum extractor.

The process of induced labour is unpredictable both in terms of the time that may elapse between the decision to end the pregnancy and eventual delivery and will always include the possibility that emergency caesarean section will be necessary. Caesarean delivery itself, either elective or emergency surgery, is associated with the risks of anaesthesia, haemorrhage and post-operative complications.

The decision to allow either vaginal delivery or to perform an elective caesarean delivery is peculiar to the individual case with multiple factors to be considered including the parity of the mother, any other obstetric co-morbidities and the severity of the cardiac lesion. There is no justification for routine caesarean delivery in women with heart failure during pregnancy although many will be delivered by this means. Individualised recommendations are available from sources such as the European Society of Cardiology Guidelines on the management of cardiovascular disease during pregnancy. ${ }^{39}$

\section{The Puerperium and Long-term Management}

Attention in the immediate puerperium should be directed to avoiding fluid overload. The delivery of the placenta leads to uterine contraction and expulsion of blood from this vascular organ into the systemic circulation. Women with severe stenosed valves and those with failing ventricles may benefit from intravenous diuretic therapy at the time of delivery. The oxytocic drugs used to facilitate active management of the third stage of labour may have vasoactive properties that should be considered prior to administration; hence bolus dose oxytocin leads to vasodilatation and reflex tachycardia while any preparation containing ergometrine will have a vasoconstrictor effect on the peripheral circulation. ${ }^{5}$ In general, low-dose oxytocin is advocated and ergometrine-containing preparations avoided. Attention also needs to be paid to the prevention of infection using prophylactic antibiotics. Recent evidence suggests that prophylactic antibiotics are necessary to limit the risk of endocarditis. ${ }^{66}$ 
The matters of identifying and treating aggravating factors has already been addressed and the question of breastfeeding also requires attention in women with peripartum cardiomyopathy.

\section{Conclusion}

Pregnancy represents a window of opportunity during which women present primarily for pregnancy care while offering an opportunity to optimise the management of known medical co-morbidities, and during which time newly diagnosed disease may be first discovered. Pregnancy is an opportunity also to secure continuity of care and to plan any future pregnancies. Once diagnosed with cardiac disease, open communication and clear policies of onward referral after the pregnancy must be established. In addition the risks of future pregnancies should be considered alongside the range of contraceptive options that are both available and appropriate. The most immediate postpartum evaluation, however, should be cardiological assessment several months after parturition in order to make a diagnosis and evaluate the recovery or deterioration in cardiac function following the conclusion of the pregnancy.
1. O'Dwyer SL, Gupta M, Anthony J. Pulmonary edema in pregnancy and the puerperium: a cohort study of 53 cases. J Perinat Med 2014: epub ahead of print.

2. Saving Mothers 2011-2013: Sixth report on confidential enquiries into maternal deaths in South Africa. Short report, Republic of South Africa Department of Health, 2015

3. Say L, Chou D, Gemmill A, et al. Global causes of maternal death: a WHO systematic analysis. Lancet Glob Health 2014;2:e323-e333.

4. Kassebaum NJ, Bertozzi-Villa A, Coggeshall MS, et al. Global, regional, and national levels and causes of maternal mortality during 1990-2013: a systematic analysis for the Global Burden of Disease Study 2013. Lancet 2014:384:980-1004.

5. Moodley J. 1225 Maternal deaths in South Africa: the latest report of the NCCEMD 2005-2007. BJOG 2009:107:S56-S57.

Knight M, Kenyon S, Brocklehurst P, et al. Saving Lives,

6. Knight M, Kenyon S, Brocklehurst P, et al. Saving Lives,
Improving Mothers' Care, Lessons learned to inform future matern Improving Mothers' Care, Lessons learned to inform future maternity
care from the UK and Ireland Confidential Enquiries into Maternal Deaths and Morbidity 2009-2012, Oxford, UK: Healthcare Quality Improvement Partnership, 2014

7. Brar SS, Khan SS, Sandhu G, et al. Incidence, mortality, and racial differences in peripartum cardiomyopathy. Am I Cardia 2007; 100:302-4

8. Thaler I, Manor D, Itskovitz J, et al. Changes in uterine blood flow during human pregnancy. Am I Obstet Gynecol 1990;162:121-5.

9. Nolten WE, Ehrlich EN. Sodium and mineralocorticoids in normal pregnancy. Kidney Int 1980;18:162-72.

10. Carlin A, Alfirevic Z. Physiological changes of pregnancy and monitoring. Best Pract Res Clin Obstet Gynaecol 2008;22:801-23.

11. Dorn GW. The fuzzy logic of physiological cardiac hypertrophy. Hypertension 2007;49:962-70.

12. Belfort $\mathrm{M}$, Anthony J, Kirshon B. Respiratory function in severe gestational proteinuric hypertension: the effects of rapid volume expansion and subsequent vasodilatation with verapamil. BJOG 1991;98:964-72.

13. Young P, Johanson R. Haemodynamic, invasive and echocardiographic monitoring in the hypertensive parturient. Best Pract Res Clin Obstet Gynaecol 2001;15:605-22.

14. Belfort M, Anthony J, Kirshon B. Respiratory function in severe gestational proteinuric hypertension: the effects of rapid volume expansion and subsequent vasodilatation with verapamil. BJOG 1991;98:964-72.

15. Belfort MA, Anthony J, Saade GR. The oxygen consumption/ oxygen delivery curve in severe preeclampsia: evidence for a fixed oxygen extraction state. Am J Obstet Gynecol 1993:169:1448-55.

16. Sibai BM, Abdella TN, Anderson GD. Pregnancy outcome in 211 patients with mild chronic hypertension. Obstet Gynecol 1983;61:571-6.

17. Sibai BM, Lindheimer $\mathrm{M}$, Hauth J, et al. Risk factors for preeclampsia, abruptio placentae, and adverse neonatal outcomes among women with chronic hypertension. N Engl J Med 1998;339:667-71.

18. Seely EW, Ecker J. Chronic hypertension in pregnancy. N Eng/」 Med 2011;365:439-46.

19. Vázquez Blanco M, Roisinblit J, Grosso O, et al. Left ventricula function impairment in pregnancy-induced hypertension. Am Hypertens 2001;14:271-5.

20. Lindheimer MD, Taler SJ, Cunningham FG; American Society of Hypertension. ASH position paper: hypertension in pregnancy. J Clin Hypertens (Greenwich) 2009;11:214-25.

21. Simonneau G, Robbins IM, Beghetti M, et al. Updated clinical classification of pulmonary hypertension. J Am Coll Cardiol 2009:54:S43-S54.

22. Weiss BM, Zemp L, Seifert B, Hess OM. Outcome of pulmonary vascular disease in pregnancy: a systematic overview from 1978 through 1996. I Am Coll Cardiol 1998:31:1650-7.

23. Sharma BK, Jain S, Vasishta K. Outcome of pregnancy in Takayasu arteritis. Int I Cardiol 2000;75:S159-S162.

24. Milewicz DM, Dietz HC, Miller DC. Treatment of aortic disease in patients with Marfan syndrome. Circulation 2005;111:e150-e157.

25. Roos-Hesselink JW, Ruys TP, Stein JI, et al. Outcome of pregnancy in patients with structural or ischaemic heart disease: results of a registry of the European Society of Cardiology. Eur Heart J 2013;34:657-65.

26. Sliwa K, Libhaber E, Elliott C, et al. Spectrum of cardiac disease in maternity in a low-resource cohort in South Africa. Heart 2014:100:1967-74.

27. Hilfiker-Kleiner D, Sliwa K. Pathophysiology and epidemiology of peripartum cardiomyopathy. Nat Rev Cardiol 2014;11:364-70.

28. Sliwa K, Fett J, Elkayam U. Peripartum cardiomyopathy. Lancet 2006; $368: 687-93$

29. Ansari AA, Fett JD, Carraway RE, et al. Autoimmune mechanisms as the basis for human peripartum cardiomyopathy. Clin Rev Allergy Immunol 2002;23:301-24.

30. Patten IS, Rana S, Shahul S, et al. Cardiac angiogenic imbalance leads to peripartum cardiomyopathy. Nature 2012;485:333-8.

31. Watkins $\mathrm{H}$, Ashrafian $\mathrm{H}$, Redwood C. Inherited cardiomyopathies. N Eng/ J Med 2011:364:1643-56

32. Autore $\mathrm{C}$, Conte MR, Piccininno M, et al. Risk associated with pregnancy in hypertrophic cardiomyopathy. I Am Coll Cardiol 2002;40:1864-9.

33. Krul SP, van der Smagt JJ, van den Berg MP, et al. Systematic review of pregnancy in women with inherited cardiomyopathies. Eur J Heart Fail 2011;13:584-94.

34. Basso C, Corrado D, Marcus Fl, et al. Arrhythmogenic right ventricular cardiomyopathy. Lancet 2009;373:1289-300.

35. Kallwellis-Opara A, Staudt A, Trimpert C, et al. Autoimmunological features in inflammatory cardiomyopathy. Clin Res Cardiol 2007:96:469-80.

36. Moore J, Baldisseri MR. Amniotic fluid embolism. Crit Care Med 2005; 33:S279-S285

37. Sliwa K, Johnson MR, Zilla P, Roos-Hesselink JW. Management of valvular disease in pregnancy: a global perspective. Eur Heart J 2015;36:1078-89.

38. Engelfriet $P, B$ Boersma $E$, Oechslin $E$, et al. The spectrum of adult congenital heart disease in Europe: morbidity and mortality in a 5 year follow-up period. Eur Heart 2005;26:2325-33.

39. European Society of Gynecology (ESG), Association for European Paediatric Cardiology (AEPC), German Society for Gender Medicine (DGesGM), et al. ESC Guidelines on the management of cardiovascular diseases during pregnancy. Eur Heart I 2011;32:3147-97.

40. Jensen D, Webb KA, O'Donnell DE. Chemical and mechanica adaptations of the respiratory system at rest and during exercise in human pregnancy. Appl Physiol Nutr Metab 2007;32:1239-50.

41. Fett JD, Validation of a self-test for early diagnosis of heart failure in peripartum cardiomyopathy. Crit Pathw Cardiol 2011:10:44-5.

42. Hytten F, Chamberlain G, Clinical physiology in obstetrics. Boston, MA: Blackwell Scientific Publications, 1980.

43. Shotan A, Ostrzega E, Mehra A, et al. Incidence of arrhythmias in normal pregnancy and relation to palpitations, dizziness, and syncope. Am J Cardiol 1997;79:1061-4.

44. Nelson-Piercy C, Chakravarti S, Cardiac disease and pregnancy. Anaesthes Intensive Care 2007;8:312-6.

45. Fisher JD, New York Heart Association Classification. Arch Int
Med 1972;129:836-6.

46. Thorne S, Nelson-Piercy C, MacGregor A, et al. Pregnancy and contraception in heart disease and pulmonary arterial hypertension. J Fam Plann Reprod Health Care 2006;32:75.

47. Catanzarite VA, Willms D, Adult respiratory distress syndrome in pregnancy: report of three cases and review of the literature. Obstet Gynecol Surv 1997;52:381-92.

48. Soubra SH, Guntupalli KK. Critical illness in pregnancy: an overview. Crit Care Med 2005;33:S248-S255.

49. Anthony, J., R. Johanson, and J. Dommisse, Critical care management of severe pre-eclampsia. Fetal Matern Med Rev 1994;6:219-29.

50. Sliwa K, Anthony J. Cardiac Drugs in Pregnancy. London, UK: Springer, 2014.

51. ter Maaten JM, Dunning AM, Valente MA, et al. Diuretic response in acute heart failure-an analysis from ASCEND HF. Am Heart J 2015;170:313-21

52. Belfort M, Akovic K, Anthony J, et al. The effect of acute volume expansion and vasodilatation with verapamil on uterine and umbilical artery Doppler indices in severe preeclampsia. J Clin Ultrasound 1994;22:317-25.

53. Clark SL, Greenspoon JS, Aldahl D, Phelan JP. Severe preeclampsia with persistent oliguria: management of hemodynamic subsets. Am J Obstet Gynecol 1986;154:490-4.

54. Gilbert WM, Towner DR, Field NT, Anthony J. The safety and utility of pulmonary artery catheterization in severe preeclampsia and eclampsia. Am J obstet Gynecol 2000;182:1397-403

55. Lüscher TF, An update on heart failure and peripheral arterial disease. Eur Heart J 2015;36:885-7.

56. Irani RA, Xia Y. The functional role of the renin-angiotensin system in pregnancy and preeclampsia. Placenta 2008;29:763-71.

57. Shotan A, Widerhorn $\rfloor$, Hurst A, Elkayam U. Risks of angiotensin-converting enzyme inhibition during pregnancy: experimental and clinical evidence, potential mechanisms, and recommendations for use. The American Journal of Medicine 1994;96:451-6.

58. Packer M, Bristow MR, Cohn JN, et al. The effect of carvedilol on morbidity and mortality in patients with chronic heart failure. N Eng/ I Med 1996;334:1349-55.

59. Swan L, Lupton M, Anthony J, et al. Controversies in pregnancy and congenital heart disease. Congenit Heart Dis 2006:1:27-34.

60. Pitt B, Zannad F, Remme WJ, et al. The effect of spironolactone on morbidity and mortality in patients with severe heart failure. N EngI J Med 1999:341:709-17.

61. Haghikia A, Podewski E, Berliner D, et al. Rationale and design of a randomized, controlled multicentre clinical trial to evaluate the effect of bromocriptine on left ventricular function in women with peripartum cardiomyopathy, Clin Res Cardiol 2015;1-7.

62. Dickstein K, Cohen-Solal A, Filippatos G, et al. ESC Guidelines for the diagnosis and treatment of acute and chronic heart failure 2008. Eur J Heart Fail 2008;10:933-89.

63. Learoyd DL, Fung HY, MCGregor AM. Postpartum thyroid dysfunction. Thyroid 1992;2:73-80.

64. Ageno W, Squizzato A, Garcia D, Imberti D, et al. Epidemiology and risk factors of venous thromboembolism. Semin Thromb Hemost 2006;32:651-8.

65. Bohlmann MK, Rath W. Medical prevention and treatmen of postpartum hemorrhage: a comparison of different guidelines. Arch Gynecol Obstet 2014;289:555-67.

66. Dayer MJ, Jones S, Prendergast B, et al. Incidence of infective endocarditis in England, 2000-13: a secular trend, interrupted time-series analysis. Lancet 2015;385:1219-28. 